\title{
Führung ist nicht gleich Führung ...
}

\section{Johannes Rüegg-Stürm}

Prof. Dr. oec., geschäftsführender Direktor des IfB der HSG. Arbeitsschwerpunkte: integrierendes Management, Management von strategischem Wandel, organisationale Wandel- und Erneuerungsfähigkeit, neue Führungs- und Organisationsformen, Prozessmanagement, systemisch-konstruktivistische Organisations- und Führungsforschung, Management von Health-Care-Organisationen

Bisher in dieser Reihe in loser Folge erschienene Artikel in SÄZ 23, 28 und 45 (alle 2007) sowie 7 und 14/2008 auf www.saez.ch.

Forschungsprogramm HealthCare Excellence

Silke Bucher, David Kurz, Jürgen Merz, Prof. Dr. Johannes RüeggStürm, Harald Tuckermann,

Dr. med. Jürg Unger-Köppel, Widar von Arx

Institut für Betriebswirtschaft, St. Gallen
Korrespondenz:

Prof. Dr. oec. Johannes Rüegg-Stürm Institut für Betriebswirtschaft

Dufourstrasse 40a

CH-9000 St. Gallen

Tel. 0712242323

Fax 0712242355

johannes.rueegg@unisg.ch

www.healthcaremanagement.ch www.ifb.unisg.ch
Im folgenden Beitrag wird gezeigt, dass wichtige Entwicklungspotentiale, was die Führung in und von Spitälern betrifft, darin liegen, wesentlich deutlicher zwischen Fachführung und Systemführung zu unterscheiden. Dies betrifft nicht nur die alltägliche Führungsarbeit als solche, sondern auch Anforderungsprofile an diese unterschiedlichen Führungsformen. Damit wird keineswegs einer simplen Ergänzung etablierter Führungspersonen im Spital durch «professionelle Manager» das Wort geredet. Wohl aber werden sich innerhalb der Ärzteschaft, der Pflege und im betriebswirtschaftlichen Kontext neue Führungsprofile entwickeln, die in zentraler Weise auf dieser Unterscheidung in Fachführung und Systemführung aufbauen werden.

\section{Ausdifferenzierung und Integration}

Die Entwicklung des Gesundheitswesens ist durch ein lang andauerndes, kontinuierliches Wachstum geprägt. Mit diesem Wachstum ist auch eine beispiellose Spezialisierung einhergelaufen. Diese Spezialisierung betrifft nicht nur die Ausdifferenzierung in Medizin, Pflege und ökonomische Funktionen (z. B. Beschaffung, Controlling, Hotellerie, Human Resource Management, Facility Management), sondern auch die professionsinterne Spezialisierung, z.B. in über dreissig medizinische Fachgesellschaften. Beides, Wachstum und Spezialisierung, sind indessen nicht nur ein Segen, sondern führen heute auch $\mathrm{zu}$ grossen Herausforderungen im Gesundheitswesen.

Erstens öffnet sich zwischen der medizinischen Machbarkeit, was innovative Therapien betrifft, und der ökonomischen Tragfähigkeit solcher Therapien eine wachsende Schere. Die Krankheitsbilder, die heute Gegenstand medizinischer Behandlung darstellen (z. B. Aids, Alzheimer, Krebs usw.), werden immer komplexer und die entsprechenden Therapien immer teurer. Diesem Trend steht ein öffentlich finanziertes Gesundheitswesen gegenüber, dessen Mittel begrenzt sind. Daraus ergibt sich ein wachsender Effizienzdruck für die Anbieter von Gesundheitsleistungen.

Zweitens zieht jede Form von wachsender Arbeitsteilung, Spezialisierung und Professionalisierung die Notwendigkeit der Integration spezialisierter Teilleistungen nach sich. Von der zeitgerechten Integration von Expertise hängt in zentraler Weise die Effektivität und Nachhaltig-

\section{II y a gestion et gestion...}

La pression à renforcer le professionnalisme se ressent aussi à l'échelon de la gestion des hôpitaux. La remise de cliniques à des gestionnaires professionnels, à l'exemple de la Grande-Bretagne, est une expérience à considérer comme un échec. Cependant, il faut s'attendre à l'avènement d'un processus de différenciation en médecine et peut-être aussi dans les soins, à l'instar de celui qui a eu lieu dans l'industrie pharmaceutique, voilà 20 ans déjà. Sur le plan des carrières professionnelles, celle-ci différencie entre une compétence scientifique $(\rightarrow$ gestion du savoir) et une compétence de gestionnaire ( $\rightarrow$ gestion de systèmes). Cette manière de différencier les professions et d'établir une spécialisation en experts professionnels d'une part et en experts de systèmes de l'autre pourrait être reprise par les hôpitaux. Il faudra pour ce faire clarifier encore bien des choses, si ce n'est envisager un changement culturel radical dans le domaine de la gestion.

keit des Behandlungsprozesses ab. Diese Integration wird in Spitälern durch viel Selbstorganisation geleistet. Spitäler zeichnen sich über eine geradezu bewundernswerte Fähigkeit zur Selbstorganisation aus. Allerdings vertreten wir aufgrund vieler Beobachtungen aus dem Arbeitsalltag von Spitälern die These, dass genau im Bereich der interdisziplinären Integration von Fachexpertise grosse Entwicklungspotentiale und auch ein erheblicher Veränderungsbedarf bestehen.

In der Managementlehre wird die skizzierte Problematik einer systematischen und bereichsübergreifenden Integration von Spezialexpertise vor allem unter der Bezeichnung «Prozessmanagement» bearbeitet. Das Konzept Prozessmanagement adressiert die Herausforderung einer strikt kundenorientierten, kostengünstigen bereichsübergreifenden Integration spezialisierter Einzelleistungen - meistens unter Nutzung neuer Möglichkeiten der Informationstechnologie. In- 
itiativen zur Entwicklung eines systematischen Prozessmanagements sind allerdings hoch anspruchsvoll und voraussetzungsreich. Sie erfordern in der Regel neue Formen der Führung und Zusammenarbeit und damit auch neuartige Beziehungs-, Kommunikations- und Führungsmuster. Ebenfalls von grosser Bedeutung ist die Informationstechnologie, die es erlaubt, vergangene und aktuelle Diagnose- und Behandlungsdaten zeitverzugslos direkt vor Ort des Geschehens den Beteiligten zur Verfügung zu stellen.

Wenn nun bei Initiativen in Richtung einer patientenzentrierten, prozessorientierten Organisation in einem Spital absehbar wird, dass dies Auswirkungen auf die Professions- oder Klinikautonomie haben könnte, führt dies meistens unvermittelt $\mathrm{zu}$ verdecktem oder gar offenem Widerstand. Dieser Widerstand wird so begründet, dass mit prozessorientierten Organisationsformen

- erstens die Verantwortlichkeiten verwischt würden;

- zweitens die Kontinuität der Patientenbetreuung in Frage gestellt sei und

- drittens durch eine gewisse Einschränkung der Professionsautonomie auch der medizinische Fortschritt beeinträchtigt werde.

Diese Argumente sind nachvollziehbar. Der fragliche Widerstand lässt sich aber zu einem erheblichen Teil auch dadurch erklären, dass die Betroffenen nicht mit der wichtigen Unterscheidung zwischen Fachführung und Systemführung vertraut sind.

\section{Fachführung und Systemführung}

Traditionell ist der Arbeitskontext vor allem der Ärzteschaft durch die Arzt-Patienten-Interaktion gekennzeichnet. In der unmittelbaren Interaktion mit dem Patienten kann die Ärztin ihre Expertise voll zur Geltung bringen. Pflege und ökonomische Funktionen sollen demgegenüber einen Systemkontext schaffen, in dem die Arbeit der Ärztin optimal vollzogen werden kann. Mit der Unterscheidung in Interaktionskontext und Systemkontext gehen auch unterschiedliche Führungsaufgaben einher, die zum einen mit dem Begriff Fachführung und zum anderen mit dem Begriff Systemführung bezeichnet werden sollen (Abb. 1):

- Unter Fachführung verstehen wir, für ein fachlich genau abgegrenztes Gebiet der therapeutischen Wertschöpfung im Kontext einer Arzt-Plegefachperson-Patienten-Interaktion die volle Verantwortung für die Qualität (Beschaffenheit) der arbeitsteilig zu leistenden Teilaktivitäten zu übernehmen. Hierzu ist me- dizinische oder pflegerische Fachexpertise auf höchstem Niveau gefragt.

- Systemführung bedeutet demgegenüber, stabile und förderliche Kontextbedingungen zu schaffen, die es überhaupt erst erlauben, die Potentiale exzellenter Fachführung voll auszuschöpfen. Im Zentrum steht dabei vor allem die Gestaltung und Weiterentwicklung der Systemlogistik, d.h. die Gestaltung und Professionalisierung von Informations- und Materialflüssen, die Institutionalisierung geeigneter (oftmals interdisziplinärer) Foren der Fachführung und die Entwicklung hierzu förderlicher Kooperationsmuster und -kulturen. Hierzu ist Managementexpertise zur wirkungsvollen Gestaltung arbeitsteiliger kommunikativer Kontexte gefragt.

Fach- und Systemführung unterscheiden sich vor allem in der Systemabgrenzung und damit im Aufmerksamkeitshorizont:

- Fachführung dient der Innovation, kontinuierlichen Weiterentwicklung und sicheren Anwendung von professionsbasiertem Fachwissen innerhalb des Interaktionskontexts zwischen Patient und Arzt bzw. Patient und Pflegefachperson. Fachführung orientiert sich an den Professionsstandards von Medizin und Pflege.

- Systemführung kann dagegen weitgehend mit Management im Gesamtsystemkontext gleichgesetzt werden und orientiert sich an den Standards professioneller Strategie- und Organisationsarbeit.

Ein differenziertes Wahrnehmungsvermögen der Unterscheidung von Fach- und Systemführung ist heute leider erst selten anzutreffen. Damit sind folgende Schwierigkeiten verbunden:

- Diese Unterscheidung erfordert zum Teil eine Redefinition vordergründig medizinischer in organisatorische Problemstellungen. Die Festlegung von Dienstplänen oder Operationsreihenfolgen bedarf wohl eines Grundverständnisses, worum es dabei aus medizinischer Sicht grundsätzlich geht. Sie braucht sich aber keineswegs ausschliesslich an medizinischen oder pflegerischen Professionsstandards $\mathrm{zu}$ orientieren, sondern genauso am Ziel einer gesamtsystembezogen optimalen Ausschöpfung knapper Infrastrukturen und Ressourcen. Damit sind organisatorische und logistische Fähigkeiten gefragt.

- Vor diesem Hintergrund besteht zwischen dem weit gefassten Führungsanspruch von Chefärzten und dem tatsächlichen, aus nachvollziehbaren Gründen oft viel enger gefass- 


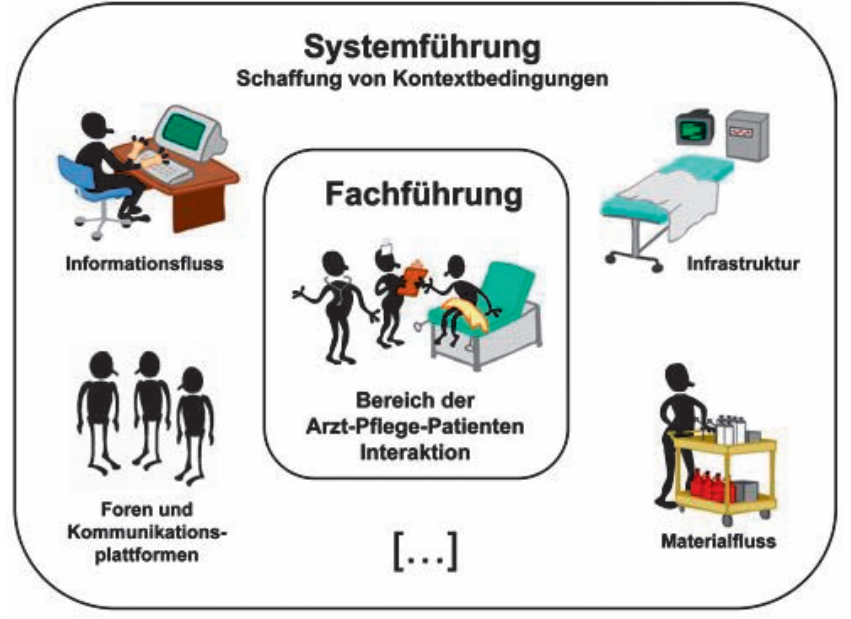

Fach- und Systemführung.

ten, auf medizinische (oder personelle) Fragestellungen eingeschränkten Fokus ihrer Gestaltungsarbeit eine problematische Asymmetrie.

- Zudem wird teilweise - wenn man z.B. Berufungsverfahren für Chefarztstellen beobachtet - immer noch unterstellt, dass eine Zunahme der Kompetenz im Bereich der Fachführung naturgemäss auch mit einer Zunahme der Kompetenz im Bereich der Systemführung verbunden sei. Diese Prämisse erweist sich aufgrund der wachsenden Ausdifferenzierung und Spezialisierung medizinischen, pflegerischen und ökonomischen Fachwissens zunehmend als unhaltbar. Aus einer sorgfältigen Unterscheidung von Fachund Systemführung ergäben sich allerdings neue Berufsprofile und veränderte Berufungsverfahren, was je nach gewachsener Professionsidentität $\mathrm{zu}$ einem mehr oder weniger schmerzhaften Prozess der Entwicklung neuer Rollen- und Führungsverständnisse führen dürfte.

Zusammenfassend empfiehlt es sich, bei einer integrativen Spitalentwicklung rechtzeitig einen Klärungsprozess einzuleiten, in dem gemeinsam für ein bestimmtes Spital spezifiziert wird, was genau Fachführung und was Systemführung beinhaltet und welche Rollenanforderungen sich daraus ergeben. Dies bildet eine wichtige Voraussetzung, um Fragen der strategischen Positionierung in der Spitalumwelt, der Prozessoptimierung und der strategischen Führungs-, Personal- und
Kulturentwicklung wechselseitig aufeinander bezogen bearbeiten zu können.

\section{Delegation an «Manager» - ein Irrweg}

Aufgabenstellungen der Systemführung im medizinischen und pflegerischen Kontext von Spitälern können allerdings nicht einfach an bewährte Führungskräfte aus Industrie und öffentlicher Verwaltung delegiert werden. Ein solides Verständnis für die Eigenheiten der Wertschöpfung von Ärzteschaft und Pflege ist unerlässliche Voraussetzung einer professionellen Systemführung. Um diese Forderung erfüllen zu können, sind zwei Optionen denkbar:

- Erfahrene Persönlichkeiten aus Ärzteschaft und Pflege eignen sich Grundkenntnisse im Bereich des Managements an und reservieren einen substantiellen Teil ihrer Arbeitszeit für die Wahrnehmung von Aufgaben der Systemführung.

- Personen mit ökonomischer Grundausbildung erarbeiten sich fundierte Kenntnisse zur Form der medizinischen und pflegerischen Wertschöpfung. Dabei geht es unserer Meinung noch nicht um die Aneignung von Professionswissen im engeren Sinne (z.B. Grundlagen der Biochemie), sondern um ein reflektiertes Verständnis der genuinen Bedingungen, Herausforderungen und routinisierten Praktiken medizinischer oder pflegerischer Arbeit mit und am Patienten.

Beide Optionen sind gleichwertig. Entscheidend ist es diesbezüglich, auf die Neigungen und Stärken bewährter Mitarbeitender abzustellen.

Wenn in diesem Beitrag eine gezielte Differenzierung von Führung in Fach- und Systemführung postuliert worden ist, entspricht dies nicht einer weltfremden Spekulation. Vielmehr ist mit dieser Ausdifferenzierung ein Prozess verbunden, der in durchaus vergleichbaren Expertenorganisationen, wie z. B. in der Pharmaindustrie, seit bald zwanzig Jahren Realität ist. Zudem ist in der Wirtschaft seit Jahrzehnten bekannt, dass es ausgesprochen problematisch sein kann, den besten Verkäufer zum Leiter Verkauf oder den besten Forscher zum Leiter Forschung und Entwicklung zu befördern. Ähnliches müsste in Spitälern bei der Spezifikation des Anforderungsprofils von Führungspositionen und insbesondere bei der Ernennung von Chefärztinnen und Chefärzten vermehrt bedacht werden. 